\title{
FACTORES ASOCIADOS A LA REALIZACIÓN DE ACTIVIDADES DE AUTOCUIDADO EN PACIENTES DIABÉTICOS EN TRES HOSPITALES DE UCAYALI
}

\author{
FACTORS ASSOCIATED WITH THE PERFORMANCE OF SELF-CARE ACTIVITIES IN DIABETIC \\ PATIENTS IN THREE UCAYALI HOSPITALS

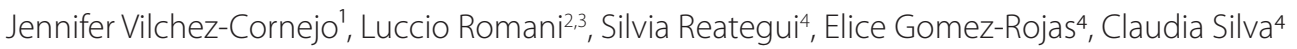

\begin{abstract}
RESUMEN
Objetivo: Determinar cuáles son los factores asociados a la realización de actividades de autocuidado en pacientes diabéticos en tres hospitales de la región de Ucayali durante el 2017. Métodos: Estudio transversal analítico, realizado en tres hospitales en Ucayali en el 2017. La encuesta autoadministrada contó con tres secciones que evaluaban las características generales de los pacientes, los conocimientos a través del Diabetes Knowledge Questionnaire 24 (DKQ-24), la actitud del paciente frente a su enfermedad mediante la Diabetes Attitude Scale (DAS-3) y Summary of Diabetes Self Care Activities Measure (SDSCA) para medir las prácticas de autocuidado. Resultados: La población de estudio estuvo conformada por 572 pacientes, el 50,87\% correspondía al sexo femenino, la mediana de la edad fue de 47 años, el 37,57\% tenía grado universitario. Se presentaron actividades de autocuidado inadecuadas con respecto al control glucémico y ejercicio con $83,87 \%$ y $77,87 \%$ respectivamente. Se observó que quienes contaron con un adecuado conocimiento y tuvieron un control estricto contaron con una adecuada adherencia respecto a la dieta de los pacientes diabéticos. Con respecto a realizar ejercicio se pudo determinar que existía una correlación negativa respecto al tiempo de enfermedad del paciente. Asimismo, se determinó que los pacientes con estudios secundarios, técnicos y universitarios presentaban una correlación negativa respecto al control glucémico. Conclusión: Más de la mitad de los pacientes realizaban actividades de autocuidado inadecuados. Además, un adecuado nivel de conocimiento y un control estricto sobre su enfermedad influencian en la adherencia a una buena dieta del paciente.
\end{abstract}

Palabras clave: Diabetes Mellitus; Autocuidado; Conocimientos; Actitudes y práctica en salud (fuente: DeCS BIREME).

\begin{abstract}
Objective: To determine which are the factors associated with self-care activities in diabetic patients in three hospitals in the Ucayali region during 2017. Methods: Analytical cross-sectional study, carried out in three hospitals in Ucayali in 2017. The survey self-administered had three sections that assess the general characteristics of the patients, knowledge through the diabetes knowledge questionnaire 24 (DKQ-24), the patient's attitude towards their disease using the diabetes attitude scale (DAS - 3) and Summary of Diabetes Self-Care Activities Measurement (SDSCA) to measure self-care practices. Results: The study population consisted of 572 patients, $50.87 \%$ were female, the median age was 47 years old, $37.57 \%$ had a university degree. Inadequate self-care activities with respect to glycemic control and exercise were performed with $83.87 \%$ and $77.87 \%$ respectively. We consider that those who have adequate knowledge and have strict control to have adequate adherence to the diet of diabetic patients. Regarding exercise, it was possible to determine that there is a negative correlation with respect to the patient's illness time. Likewise, it was determined that patients with secondary, technical and university studies had a negative correlation with respect to glycemic control. Conclusion: More than half of the patients carried out inadequate self-care activities. Furthermore, an adequate level of knowledge and strict control over their disease influence the patient's adherence to a good diet.
\end{abstract}

Key words: Diabetes mellitus; Self-care; Health knowledge; Attitudes; Practice (source: MeSH NLM).

${ }^{1}$ Red Asistencial Ucayali, Hospital EsSalud II Pucallpa, Ucayali-Perú.

2 Facultad de Medicina Humana, Universidad de San Martín de Porres, Chiclayo-Perú.

${ }^{3}$ Sociedad Científica de Estudiantes de Medicina Veritas, Lima-Perú.

${ }^{4}$ Facultad de Medicina Humana, Universidad Nacional de Ucayali, Ucayali-Perú.

Citar como: Jennifer Vilchez-Cornejo, Luccio Romani, Silvia Reategui, Elice Gomez-Rojas, Claudia Silva. Factores asociados a la realización de actividades de autocuidado en pacientes diabéticos en tres hospitales de Ucayali. Rev. Fac. Med. Hum. Abril 2020; 20(2):254-260. DOI 10.25176/ RFMH.v20i2.2902

Artículo publicado por la Revista de la Facultad de Medicina Humana de la Universidad Ricardo Palma. Es un artículo de acceso abierto, distribuído bajo los términos de la Licencia Creative Commons: Creative Commons Attribution 4.0 International, CC BY 4.0 (https://creativecommons.org/licenses/by/4.0/), que permite el uso no comercial, distribución y reproducción en cualquier medio, siempre que la obra original sea debidamente citada. Para uso comercial, por favor póngase en contacto con revista.medicina@urp.pe 


\section{INTRODUCCIÓN}

La diabetes mellitus tipo II (DM2) actualmente se considera un problema de salud pública mundial. Se estima que a nivel global lo casos de diabetes alcanzarán los 592 millones en el 2035, afectando al 8,8\% de la población mundial(1). A nivel mundial, se estima que 425 millones de adultos padecen de diabetes mellitus (DM) ${ }^{(2)}$. Se espera que aumento en la prevalencia de DM produzca un aumento en el número de enfermedades crónicas y agudas en la población, afectando directamente en la calidad de vida, la demanda en servicios de salud y la economía a nivel global ${ }^{(1,2)}$.

En América Central y del Sur, más de 26 millones de personas padecen de diabetes. Se estima que, el número de pacientes diabéticos puede aumentar a 693 millones para el 2045. En la actualidad existen reportes de hasta 352 millones de personas con alteración de la tolerancia a la glucosa, presentando éstas, un alto riesgo de desarrollar DM. Asimismo, se reporta que los países con ingresos bajos y medios soportan casi el $80 \%$ de la carga de DM a nivel mundial ${ }^{(3,4)}$. En el Perú, se han registrado cerca de 24 mil casos para el 2018, siendo cerca del 61,4\% de los diagnosticados con DM 2 eran mujeres ${ }^{(5)}$.

La carga de diabetes disminuye los presupuestos nacionales de salud, reduce la productividad, frena el crecimiento económico, genera elevados gastos en hogares vulnerables; además genera una gran carga humana, la cual se caracteriza por una mortalidad prematura y la baja calidad de vida a consecuencia de las complicaciones de la enfermedad, generando un impacto económico importante tanto para los países, como para los sistemas de salud(6).

Se ha demostrado que la educación en DM de forma continua, es una herramienta fundamental, tanto para la población vulnerable y con factores de riesgo, como para quienes ya sufren de dicha condición ${ }^{(6)}$. Educar e informar al paciente con DM, medidas de autocuidado y manejo de la enfermedad, es un abordaje eficaz para lograr adecuada adherencia al tratamiento, cuyo propósito es el controlar los valores glucémicos y disminuir la posibilidad de presentar comorbilidades y complicaciones tanto agudas como crónicas ${ }^{(7,8)}$.

El autocuidado se define como el conjunto de acciones intencionadas que realiza la persona para controlar los factores que pueden comprometer su vida y desarrollo posterior ${ }^{(9)}$. La Asociación Americana de Diabetes ha planteado al autocuidado como parte del manejo inicial y del seguimiento en pacientes con DM, y ha sido incorporado en las guías de práctica clínica, debido a su eficacia, mejoría de sobrevida, disminución de complicaciones y cumplimiento de las metas terapéuticas ${ }^{(7,8)}$.

Por lo que, la presente investigación cuenta como objetivo el de determinar los factores asociados a la realización de actividades de autocuidado en pacientes diabéticos en tres hospitales de la región de Ucayali durante el 2017.

\section{MÉTODOS}

Se realizó un estudio transversal analítico, realizado durante el 2017 en tres sedes hospitalarias de la región de Ucayali los cuales corresponden al Seguro Social de Salud (hospital II EsSalud Pucallpa) y al Ministerio de Salud (hospital Amazónico y hospital Regional de Pucallpa).

\section{Población y muestra}

La población inicial de estudio estuvo conformada por 4 059 pacientes diagnosticados con DM 2 distribuidos en 2531 pacientes del hospital EsSalud II de Pucallpa, 522 del hospital Amazónico y 1006 pacientes del hospital Regional, quienes acudieron a los nosocomios para su control/tratamiento y/o hospitalización. Se calculó un tamaño muestral de 572 participantes, utilizando el programa estadístico EPIDAT con un nivel de confianza del $95 \%$, un margen de error del $5 \%$ y una prevalencia del $50 \%(0,5)$. Se realizó un muestreo por conveniencia en donde se procedió a encuestar a los participantes hasta alcanzar el tamaño muestral calculado previamente.

Se consideraron dentro del estudio a los pacientes diagnosticados con DM 2, dentro de los servicios de hospitalización, emergencia y/o consultorio externo, quienes se encontraban estables hemodinámicamente aceptando participar en el estudio firmando su consentimiento informado. Se excluyó a los pacientes imposibilitados de completar la encuesta y/o quienes tuvieron que ser referidos de otros centros fuera de la región de Ucayali.

\section{Procedimiento y variables}

Se aplicó una encuesta anónima autoadministrada que abarcó a las características generales de los pacientes (edad, sexo, estado civil y grado de instrucción), conocimientos sobre DM 2, la actitud del paciente frente a la DM 2 y actividades de autocuidado.

Se empleó el Summary of Diabetes Self Care Activities Measure $(S D S C A)^{(10)}$ para medir las prácticas de autocuidado en DM, su versión adaptada al español(11) cuenta con un coeficiente de Cronbach de 0,764. $\mathrm{El}$ instrumento evalúa tres factores principales correspondientes a la dieta, ejercicio y el control de la glucemia, siendo la escala de respuesta mediante una puntuación tipo Likert que va de 0 a 7 puntos en función al número de días en la última semana ${ }^{(11)}$. Para la interpretación de los resultados se delimitó como punto de corte de 3,5 , siendo consideradas las respuestas menores o iguales a esta como inadecuadas.

El nivel de conocimientos de diabetes fue evaluado a través Diabetes Knowledge Questionnaire 24 (DKQ $24^{(12)}$, versión reducida y en español del DKQ-60 ${ }^{(13)}$. Cuya consistencia interna correspondió a un Alpha de Cronbach 
de $0,78^{(13)}$. El actual instrumento consta con 24 ítems con alternativas de 'sí,'no'y'no sé', otorgándose un punto a las respuestas correctas y cero a las incorrectas. El cuestionario cuenta con tres secciones que definen conocimientos básicos sobre la enfermedad, control de la glucemia y prevención de las complicaciones. Para su evaluación y análisis la puntuación global y correspondiente a cada sección se calculó sumando el puntaje de los veinticuatro reactivos, siendo los puntajes más altos quienes indicaron un mayor nivel de conocimiento sobre la $\mathrm{DM}^{(12)}$. Para fines del presente estudio, se consideró un buen conocimiento cuando se obtuvo más del $50 \%$ de respuestas correctas (mayor a 12 puntos).

Las actitudes y motivaciones relacionadas a los pacientes se midieron a través de la Diabetes Attitude Scale (DAS - 3) $)^{(14)}$, en su versión adaptada al español ${ }^{(15)}$ en una población similar, cuya consistencia interna contaba con un coeficiente de alfa de Cronbach de $0,74^{(15)}$. Este compuesto cuenta con cinco subescalas las cuales definen la necesidad de entrenamiento, percepción de la gravedad de diabetes mellitus, la valoración del control estricto, la valoración del impacto psicosocial de la diabetes mellitus y la autonomía del paciente. Así mismo, consta de 33 ítems mediante una escala tipo Likert con una puntuación máxima de 5 y una mínima de 1, correspondiente a "totalmente de acuerdo" (5 puntos), "de acuerdo" (4 puntos), "indiferente" (3 puntos), "en desacuerdo" (2 puntos) o "totalmente en desacuerdo" (1 punto). Sin embargo, los valores de las puntuaciones fueron inversas (Ítems 2; 3; 7; 11; 13; 15; 16; 23; 26 y 28). Las encuestas que no fueron contestadas en más el $50 \%$ de las preguntas en una de las subescalas fueron consideradas como nulas ${ }^{(15)}$. Además, durante nuestra investigación se tomó como punto de corte, una puntuación menor o igual a 3 como inadecuado para cada sección.

\section{Análisis estadístico}

Culminada la recolección de datos de los participantes, los datos fueron registrados en una hoja de cálculo del programa Microsoft Office Excel 2013. Siendo analizados a través del paquete estadístico STATA versión 15. Para la estadística descriptiva de las variables cuantitativas se aplicó el test de Sknewness Kurtosis(Sktest) para encontrar la normalidad de la distribución de los resultados, las variables que presentaron una distribución normal son expresadas en media y desviación estándar ( $p>0,05)$ y las que no, en mediana y rangos intercuartílicos. Los resultados se presentaron en tablas y gráficos, expresando los datos obtenidos en expresión nominal y porcentual.

Finalmente, con respecto a la estadística inferencial se tomó como variables dependientes a los resultados de las subescalas de la SDSCA (dieta, ejercicio y control de la glucemia) y como variables independientes a edad, grado de instrucción, nivel de conocimientos sobre la enfermedad obtenido del DKQ-24 y el puntaje total del
DAS-3. Para el análisis se usaron modelos generalizados aplicando razones de prevalencia cruda (RPc) y ajustada (RPa) y sus intervalos de confianza al 95\% con respecto a las variables de autocuidado, considerándose como significativo y valor $p<0,05$.

\section{Consideraciones éticas}

La recolección de la información se realizó mediante un encuestador el cual solicitaba permiso a los médicos asistenciales de los nosocomios y posteriormente el consentimiento informado de los propios pacientes. Se respetó las implicaciones éticas derivadas de acuerdos internacionales en la materia, contenidas en el Acuerdo de Helsinki. Así mismo, el protocolo del presente estudio fue aprobado por el Comité de Ética de la Universidad Nacional de Ucayali. Además, se empleó un consentimiento informado que fue firmado por cada uno de los participantes de la investigación.

\section{RESULTADOS}

La población de estudio estuvo conformada por 572 pacientes, siendo el 50,87\% (291) de sexo femenino, la mediana de la edad fue de 47 años (rangos intercuartílicos: 35 - 56). Según el estado civil 44,93\% refirió estar casado, $30,42 \%$ (174) estar conviviendo con su pareja, 16,26\% (93) soltero y $8,39 \%$ (48) refirieron ser viudos. Según el grado de instrucción reportado el 37,57\% (215) de los pacientes refirieron tener un nivel universitario, 30,97\% (177) secundario, 22,90\% (131) técnico, 6,64\% (38) primario y $1,92 \%$ (11) refirió no tener instrucción educativa alguna. Con respecto al tipo de institución hospitalaria, el 37,41\% (214) de la población fueron atendidos en alguna sede hospitalaria del ministerio de salud y el $62,59 \%$ (358) en EsSalud.

Dentro de las características clínicas de la población estudiada, se mostró una mediana del tiempo de enfermedad de 3 años (rangos intercuartílicos: 1 - 7). El tipo de tratamiento más usado fueron los antidiabéticos orales con $70,80 \%$ (405), seguido del tratamiento con insulina con 19,41\% (111) y la combinación de ambos con 9,79\% (56), reportándose un seguimiento de tratamiento regular en el 83,69\% (479) de la población estudiada. Dentro de las comorbilidades reportadas el 22,42\% (128) pacientes refirieron presentar hipertensión arterial (HTA), 9,81\% (56) obesidad 4,90\% (28) asma, 3,50\% cardiopatías y $1,93 \%$ (11) EPOC, Asimismo el 57,44\% de los pacientes refirieron no presentar comorbilidades.

Dentro de las dimensiones de las actividades de autocuidado de la SDSCA, se encontró que más de la mitad de los pacientes realizaban actividades de autocuidado inadecuados respecto al control glucémico y ejercicio, llegando a $83,87 \%$ (479) en el control de la glucémico y $77,87 \%$ (446) en el ejercicio; con respecto al autocuidado en la dieta el 63,64\% (364) de los pacientes mostró tener 
actividades de autocuidado adecuados (Figura 1). Según las dimensiones de las actitudes frente a la diabetes, reportadas a través de la DAS-3, se presentó con mayor frecuencia las actitudes inadecuadas frente a la diabetes, llegando a 63,64\% (364) en la autonomía de la diabetes, $69,93 \%$ (400) en la valoración del impacto psicosocial, $44,06 \%$ (252) en la valoración del control estricto, 51,59\% (295) en la percepción de la gravedad y 69,23\% (396) en la necesidad de entrenamiento (Figura 2).

Dentro de las dimensiones de la escala de conocimientos sobre diabetes (DQK-24), se encontró que el 65,56\% (375) de los participantes presentaba un nivel de conocimientos inadecuado, reportándose una puntuación media de 4,63 $\pm 1,81$ en los conocimientos básicos de la enfermedad, 2,59 \pm 1,31 en el control glucémico, y 3,78 \pm 1,47 en la prevención de las complicaciones, teniendo una puntuación de $11 \pm 3,14$ como media de la puntuación total (Tabla 1).

Con respecto a la estadística inferencial quienes contaron con un adecuado nivel de conocimientos (RPa: 1,31;
IC 95\%: 1,01-1,48) predisponían a una adherencia respecto a la dieta. Del mismo modo, presentaron una correlación negativa siendo las actitudes del paciente frente a su enfermedad (RPa: 0,88; IC 95\%: 0,85-0,92) y los grados de estudios primarios (RPa: 0,84; IC 95\%: 0,98 - 0,99), estudios técnicos (RPa: 0,64; IC 95\%: 0,49-0,76) y estudios universitarios (RPa: 0,58; IC 95\%: 0,51 - 0,80) estadísticamente significativos en la dieta (Tabla 2).

Con respecto a la adherencia a realizar ejercicio se pudo determinar que existía una correlación negativa en base al tiempo de enfermedad del paciente (RPa:0,92 IC 95\%:0,86 - 0,98) además de la actitud que presentaba el paciente predisponía hasta en un 21\% (RPa: 1,21; IC 95\%: 1,07 $1,37)$ respecto a la realización del ejercicio. Finalmente, se pudo determinar una asociación estadísticamente significativa con respecto al grado de instrucción en aquellos con estudios secundarios (RPa: 0,20; IC 95\%: 0,08 - 0,55), estudios técnicos (RPa: 0,32; IC 95\%: 0,12 - 0,82) y estudios universitarios (RPa: 0,39; IC 95\%: 0,16 $0,93)$ presentando una correlación negativa respecto a la adherencia a un adecuado control glucémico (Tabla 2).

\section{ACTIVIDADES DE AUTOCUIDADO}

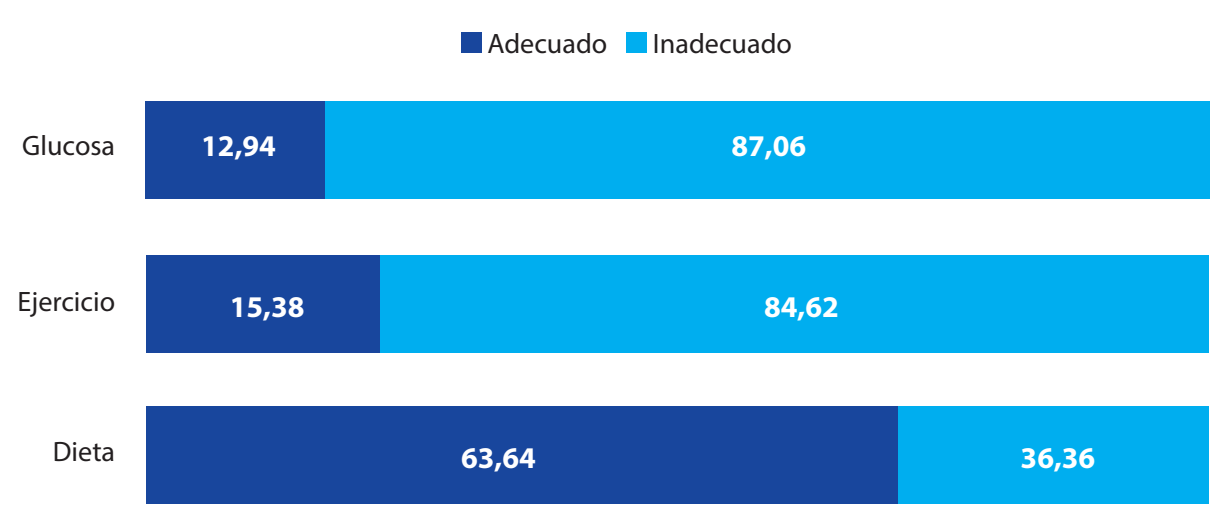

Figura 1. Dimensiones de las actividades de autocuidado en pacientes diabéticos atendidos en tres hospitales de la Amazonía peruana.

\section{ACTITUDES FRENTE A LA DIABETES}

\begin{tabular}{|c|c|c|}
\hline Autonomía del paciente & 36,36 & 63,64 \\
\hline Valoración del impacto psicosocial & 30,07 & 69,93 \\
\hline Valoración del control estricto & 55,94 & 44,06 \\
\hline Percerpción de la gravedad & 48,23 & 51,57 \\
\hline Necesidad de entrenamiento & 30,77 & 69,23 \\
\hline
\end{tabular}

Figura 2. Dimensiones de las actitudes frente a la diabetes en pacientes diabéticos atendidos en tres hospitales de la Amazonía peruana. 
Tabla 1. Dimensiones de la escala de conocimientos sobre diabetes (DQK-24) en pacientes diabéticos atendidos en tres hospitales de la Amazonía peruana.

\begin{tabular}{lccccc} 
& Media & $\begin{array}{c}\text { Desviación } \\
\text { estándar }\end{array}$ & Rang \\
& & 4,63 & 1,81 & 0 & 9 \\
\hline Inferior & Superior \\
Conocimientos básicos de la enfermedad & 2,59 & 1,31 & 0 & 7 \\
Prevención de complicaciones & 3,78 & 1,47 & 0 & 7 \\
Puntaje total & 11 & 3,14 & 1 & 20 \\
\hline
\end{tabular}

Tabla 2. Análisis crudo y ajustado de los factores asociados de las actividades de autocuidado.

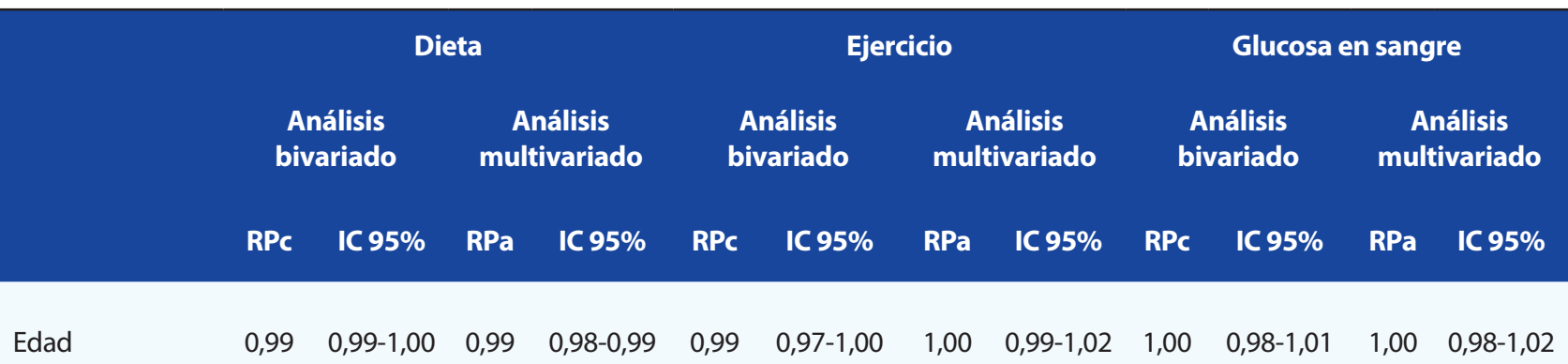

\section{Grado de instrucción}

\begin{tabular}{|c|c|c|c|c|c|c|c|c|c|c|c|c|}
\hline Ninguno & Ref. & & Ref. & & Ref. & & Ref. & & Ref. & & Ref. & \\
\hline $\begin{array}{l}\text { Estudios } \\
\text { primarios }\end{array}$ & 0,84 & 0,65-1,09 & 0,84 & $0,98-0,99$ & 1,30 & $0,33-5,17$ & 1,31 & $0,38-4,53$ & 0,80 & $0,31-2,01$ & 0,80 & $0,31-2,10$ \\
\hline $\begin{array}{l}\text { Estudios } \\
\text { secundarios }\end{array}$ & 0,75 & $0,60-0,92$ & 0,61 & $0,66-1,08$ & 0,40 & $0,10-1,57$ & 0,45 & $0,13-1,54$ & 0,19 & $0,07-0,48$ & 0,20 & $0,08-0,55$ \\
\hline $\begin{array}{l}\text { Estudios } \\
\text { técnicos }\end{array}$ & 0,68 & $0,54-0,86$ & 0,64 & $0,49-0,76$ & 0,97 & $0,26-3,57$ & 0,85 & $0,26-2,72$ & 0,34 & $0,14-0,83$ & 0,32 & $0,12-0,82$ \\
\hline $\begin{array}{l}\text { Estudios } \\
\text { universitarios }\end{array}$ & 0,63 & $0,51-0,79$ & 0,58 & $0,51-0,80$ & 1,05 & $0,29-3,79$ & 0,99 & $0,32-3,08$ & 0,40 & $0,17-0,93$ & 0,39 & $0,16-0,93$ \\
\hline $\begin{array}{l}\text { Tiempo de } \\
\text { enfermedad }\end{array}$ & 1,01 & $1,01-1,02$ & 1,02 & $0,46-0,72$ & 0,91 & $0,86-0,97$ & 0,92 & $0,86-0,98$ & 0,96 & $0,90-1,03$ & 0,96 & $0,89-1,03$ \\
\hline $\begin{array}{l}\text { Nivel de } \\
\text { conocimientos* }\end{array}$ & 1,38 & $1,43-1,79$ & 1,31 & $1,01-1,48$ & 0,80 & $0,52-1,22$ & 1,26 & $0,80-2,00$ & 0,66 & $0,40-1,08$ & 0,88 & $0,50-1,57$ \\
\hline Actitudes** & 0,85 & $0,82-0,88$ & 0,88 & $0,85-0,92$ & 1,25 & $1,12-1,38$ & 1,21 & $1,07-1,37$ & 1,15 & $1,03-1,28$ & 1,09 & $0,95-1,24$ \\
\hline
\end{tabular}

RPc: Razón de prevalencias, crudo / RPa: Razón de prevalencias, ajustado.

*Nivel obtenido a través de la DKQ-24 / **Puntaje total obtenido de la DAS-3. 


\section{DISCUSIÓN}

El fomento de conductas de autocuidado, entendido como una actividad iniciada de forma consciente y con un proceso de aprendizaje, apropiada a una determinada situación y centrada en un objetivo concreto ${ }^{(16)}$, constituye un elemento nuclear en el abordaje de las enfermedades crónicas de forma general, y en la DM especialmente ${ }^{(17,18)}$. En este artículo, buscamos determinar la asociación entre las características sociodemográficas, los conocimientos, actitudes y las actividades de autocuidado que tienen los pacientes con DM en el segundo nivel de atención.

Las principales comorbilidades reportadas en los pacientes fueron de quienes además contaban con HTA y la obesidad, se ha logrado determinar que la DM 2 se asocia con un riesgo sustancialmente mayor de eventos micro y macrovasculares como infartos de miocardio y accidentes cerebrovasculares ${ }^{(19)}$. Además, el desarrollo de HTA en pacientes diabéticos se debe a la aparición de proteinuria, del deterioro de la función renal y complicaciones cardiovasculares ${ }^{(20)}$. Del mismo modo, se ha determinado que los principales mecanismos para generar HTA han sido debido a la hiperinsulinemia por resistencia a la insulina estimulada por la actividad simpática y la retención renal de sodio, una expansión de volumen secundaria a niveles elevados en glucosa y por la reabsorción de sodio, seguido de una rigidez vascular secundaria a glicosilación proteica ${ }^{(20,21)}$.

La educación sobre la diabetes y el manejo del cuidado de la diabetes siguen siendo áreas de interés y preocupación para los profesionales de la salud e investigadores clínicos, incluso aunque se ha realizado una gran cantidad de investigación en esta área. Nuestros resultados muestran que el $65,56 \%$ de los participantes presentaban niveles inadecuados en cuantoal conocimientos sobrela diabetes, esto es congruente con hallazgos de otros estudios ${ }^{(22-24)}$ Tener conocimientos adecuados relacionados con la etiología de la diabetes, la sintomatología presente en los niveles altos o bajos de glucemia en sangre, manejo de heridas, y la idea errónea de que el uso regular de medicamentos es más importante que la ingesta dietética y el ejercicio en el control de la diabetes.

Más de la mitad de los pacientes diabéticos realizaban actividades de autocuidado inadecuados, resultados que resaltan la necesidad de mejorar las actividades de autocuidado. Para la realización de cambios en los estilos de vida, son necesarios enfoques innovadores en la educación y actividades de autocuidado. Se recomiendan intervenciones flexibles de autocuidado que puedan responder a un enfoque individual, único, diario y cultural(25).

La actitud frente a la enfermedad es un punto clave en la determinación patrones de comportamiento, representando una predisposición para la adopción de acciones de autocuidado, favoreciendo la reducción del estrés asociado a la enfermedad, mayor adherencia al tratamiento, mejora de la autoestima, además brinda una percepción más positiva sobre la salud ${ }^{(26)}$. Los resultados de la aplicación de la DAS-3 en nuestro estudio, reportó con mayor frecuencia las actitudes inadecuadas frente a la DM, demostrando de esta forma, la necesidad de desarrollar actividades de enseñanza y prácticas educativas de la salud, centradas en el fortalecimiento de actitud activa frente a la enfermedad, dado que esta se encuentra relacionada con la mejora del automanejo de la enfermedad, mejorando la convivencia de la persona con su condición ${ }^{(25,27,28)}$.

Un estudio similar ${ }^{(29)}$, identificó la actitud que la mayoría de los pacientes diabéticos pueden llegar a desarrollar ante su enfermedad, en contraste con nuestro estudio, donde se refleja que solo una pequeña parte de ellos se ve en la necesidad de cambiar sus hábitos alimenticios. En nuestro estudio, se pudo observar que un nivel de conocimiento adecuado, además de brindarle a los pacientes un control estricto en las recomendaciones sobre su enfermedad, conllevaba a mejora sustanciales en su dieta, esto podría deberse a que la mayoría de los pacientes que debutan con DM2 no consideran a la dieta como un hábito a mejor dentro de su nuevo estilo de vida, ya que inicialmente se estima que nivel de conocimientos que estos acarrean es deficiente ${ }^{(30)}$. El control estricto de los pacientes coincide según diversos investigadores ${ }^{(31-33)}$, en las intervenciones basadas para mejorar conducta en su alimentación, resultando estas, beneficiosas al inicio del tratamiento, sobre todo en aquellos pacientes quienes se encontraban dispuestos a realizar cambios en su dieta, lo que explicaría por qué cuando llegan a adaptarse adecuadamente cuentan con prácticas adecuadas.

Las limitaciones presentadas durante la investigación fue la tasa de rechazo de los pacientes diabéticos encuestados durante la consulta externa, hospitalización y/o emergencia, además la falta de un estudio caso control y/o longitudinal para así delimitar la causalidad entre las variables. Nuestra investigación, recomienda mejorar las actividades de autocuidados dentro de los pacientes diabéticos, reforzar los programas en los que se encuentran los pacientes diabéticos, así como buscar estrategias para realizar un acompañamiento por unidades especializadas (nutrición, psicología, endocrinología) los cuales puedan brindar mejores herramientas para contar con un óptimo autocuidado.

\section{CONCLUSIÓN}

Finalmente, se puede concluir que más de la mitad de los pacientes realizaban actividades de autocuidado inadecuados. Además, un adecuado nivel de conocimiento y un control estricto sobre su enfermedad influencian en la adherencia a una buena dieta del paciente. 
Agradecimientos: Los autores de la presente investigación desean agradecer a la universitaria Luz Vanessa Valdivia Martell por la recolección de los datos y formar parte de las versiones iniciales de la presente investigación.

Contribuciones de autoría: Los autores participaron en la génesis de la idea, diseño de proyecto, recolección e interpretación de datos, análisis de resultados y preparación del manuscrito del presente trabajo de investigación.

Financiamiento: La presente investigación se encuentra parcialmente financiada por el Fondo de Desarrollo Socioeconómico de Camisea (FOCAM) de la Facultad de Medicina Humana correspondiente a la Universidad
Nacional de Ucayali durante el año 2017.

Conflicto de interés: La presente investigación formó parte parcialmente de la tesis de pregrado de la médica cirujana Claudia Cristina Silva Bardales. Sin embargo, los autores declaran no presentar ningún conflicto de interés personal.

Recibido: 12 de marzo 2020

Aprobado: 30 de marzo 2020

Correspondencia: Jennifer Steffany Vilchez Cornejo

Dirección: Jr. Ramón Castilla 159 Mz E Lote 11, Ucayali-Perú.

Teléfono: 961506868

Correo:j.vilchezcornejo@gmail.com

\section{REFERENCIAS BIBLIOGRÁFICAS}

1. Guariguata L, Whiting DR, Hambleton I, Beagley J, Linnenkamp U, Shaw JE. Global estimates of diabetes prevalence for 2013 and projections for 2035.
Diabetes Res Clin Pract. 2014;103(2):137-49. DOl:https://doi.org/10.1016/j. Diabetes Res Clin
diabres.2013.11.002

2. Ogurtsova K, da Rocha Fernandes JD, Huang Y, Linnenkamp U, Guariguata L, Cho NH, et al. IDF diabetes atlas: global estimates for the prevalence of diabete for 2015 and 2040. Diabetes Res Clin Pract. 2017;128:40-50. DOl:https://doi. org/10.1016/j.diabres.2017.03.024

3. Organización Mundial de la Salud (OMS). Informe mundial sobre la diabetes (Internet); 2016 (citado 10 de marzo de 2020). Disponible en:https://www. who.int/diabetes/global-report/es/.

4. International Diabetes Federation (IFD) Diabetes Atlas. Octava edición 2017. Panorama mundial. 2017; c3: 40-4. Disponible en: http://fmdiabetes.org/atlasidf-2017/

5. Revilla L. Equipo Técnico Vigilancia de ENT, CDC/MINSA. Informe de Vigilancia de Diabetes al IV trimestre 2018. 2019.:

6. Hevia EV. Educación en Diabetes. Revist Médica Clínica los Condes; 2016 27(2): 271-6. DOl:https://doi.org/10.1016/j.rmclc.2016.04.016

7. American Diabetes Association. Standards of medical care in diabetes. Diabetes Care. 2016; 39(s 1): S4-S5. DOl:https://doi.org/10.2337/dc16-S003

8. Powers MA, Bardsley J, Cypress M, Duker P, Funnell MM, Fischl AH, et al. Diabetes self-management education and support in type 2 diabetes: $A$ joint position statement of the American Diabetes Association, the American Association of Diabetes Educators, and the Academy of Nutrition and Dietetics. Diabetes Care [Internet]. 2015, [Citado 28 febrero 2020]; 38(7): 137282. DOl:https://dx.doi.org/10.2337\%2Fdiaclin.34.2.70

9. Denyes $\mathrm{MJ}$, Orem DE, Bekel G. Self-Care: A Foundational Science. Nursing Science Quarterly. 2001; 14(1): 48-54. DOI:https://doi. org/10.1177/089431840101400113

10. Schmitt A, Gahr A, Hermanns N, Kulzer B, Huber J, Haak T. The Diabetes Self-Management Ouestionnaire (DSMO): development and evaluation of an instrument to assess diabetes self-care activities associated with glycaemic control. Health Qual Life Outcomes. 2013; 11: 138. DOl:https://doi. org/10.1186/1477-7525-11-138

11. Caro-Bautista J, Morilla-Herrera JC, Villa-Estrada F, Cuevas-FernándezGallego M, Lupiáñez-Pérez I, Morales-Asencio JM. Adaptación cultural a español y validación psicométrica del Summary of Diabetes Self-Care Activities measure (SDSCA) en personas con diabetes mellitus tipo 2. Aten Primaria 2016; 48(7): 458 - 67. DOl:https://doi.org/10.1016/j.aprim.2015.08.005

12. García AA, Villagomez ET, Brown SA, Kouzekanani K, Hanis CL. The Star County. Diabetes education study: Development of the Spanish-language Diabetes Knowledge Questionnaire. Diabetes Care 2001;24:16-21. DOI:https:// doi.org/10.2337/diacare.24.1.16

13. Villagomez E. Health Beliefs, Knowledge, and Metabolic Control in Diabetic Mexican American Adults. MS thesis. Houston, TX, The University of Texas Health Science Center, 1989

14. Anderson RM, Fitzgerald JT, Funnell MM, Gruppen LD. The third version of the Diabetes Attitude Scale. Diabetes Care 1998; 21: 1403-1407. DOl:https:// doi.org/10.2337/diacare.21.9.1403

15. Hernández JM, Basora J, Ansa X, Piñol JL, Millan M, Figuerola D. La versión española de la Diabetes Attitude Scale (DAS-3sp): un instrumento de medición de actitudes y motivaciones en Diabetes. Endocrinol Nutr. 2002; 49: 293-8. DOl:https://doi.org/10.1016/S1575-0922(02)74476-8

16. Mailhot $T$, Cossette $S$, Alderson M.Une analyse évolutionniste du concept d'autosoins. Rech Soins Infirm. 2013; 112: 94-106. DOl:https://doi.org/10.3917/ rsi.112.0094

17. Grupo de trabajo de la Guía de Práctica Clínica sobre Diabetes tipo 2. Guía de Práctica Clínica sobre Diabetes tipo 2 Madrid: Plan Nacional para el SNS del MSC. Agencia de Evaluación de Tecnologías Sanitarias del País Vasco; 2008.
Guías de Práctica Clínica en el SNS: OSTEBA N.o 2006/08. Disponible en:https:// portal.guiasalud.es/wp-content/uploads/2018/12/GPC_429_Diabetes_2 Osteba_compl.pdf

18. National Institute for Health and Clinical Excellence. The management of type 2 diabetes: NICE clinical guideline 87. 2009; 8-9. PIMD:21678628

19. Woodward M, Zhang X, Barzi F, et al. The effects of diabetes on the risks of major cardiovascular diseases and death in the Asia-Pacific region. Diabetes Care. 2003;26(2):360-6. DOl:https://doi.org/10.2337/diacare.26.2.360

20. Emdin CA, Rahimi K, Neal B, Callender T, Perkovic V, Patel A. Blood pressure lowering in type 2 diabetes: a systematic review and meta-analysis. JAMA. 2015;313(6):603-15. DOl:https://doi.org/10.1001/jama.2014.18574

21. De la Fuente GA, Tocora DGG, Sevillano BH, Del Valle KMP. Protocolo diagnóstico y tratamiento de la hipertensión en el síndrome metabólico y en la diabetes mellitus tipo 2. Medicine. 2019; 12(81): 4800-3. DOl:https://doi. org/10.1016/j.med.2019.06.008

22. Rankin D, Heller S, Lawton J. Understanding information and education gaps among people with type 1 diabetes. Patient Educ Couns. 2011; 83(1): 8791. DOl:https://doi.org/10.1016/j.pec.2010.04.026

23. Strine TW, Okoro CA, Chapman DP, et al. The impact of formal diabetes education on the preventive health practices and behaviours of persons with type 2 diabetes. Prev Med. 2005; 41(1):79-84. DOl:https://doi.org/10.1016/j. ypmed.2004.10.009

24. Formosa C, Muscat R. Improving Diabetes Knowledge and Self-Care Practices. J Am Podriatr Med Assoc. 2016; 106(5): 352-356. DOl:https://doi. org/10.7547/15-071

25. Funnell MM, Brown TL, Childs BP, et al. National standards for diabetes self-management education. Diabetes Care.2010; 34(suppl 1): S89-S96. DOl:https://dx.doi.org/10.2337\%2Fdc10-S089

26. Rodrigues FFL, Zanetti ML, Manoel AS, Martins TA, Sousa VD, Teixeira CRS Knowledge and attitude: important components in diabetes education. Rev Latino-Am Enfermagem. 2009;17(4):468-73. DOl:https://doi.org/10.1590/ S0104-11692009000400006

27. Roter DL, Hall JA, Merisca R, Nordstrom B, Cretin D, Svarstad B. Effectiveness of interventions to improve patient compliance: a meta-analysis. Med Care 1998;36(8):1138-61.DOl:https://doi.org/10.1097/00005650-199808000-00004

28. Sousa VD, Zauszniewski JA, Lea PJP, Davis SA. Relationships among selfcare agency, self-efficacy, self-care, and glycemic control. Res Theory Nurs Practice 2005;19(3):217-30. DOl:https://doi.org/10.1891/rtnp.2005.19.3.217

29. Carballo M; Mohammad A; Maclean EC; Khatoon N; Waheedi M; Abraham $\mathrm{S}$. Knowledge, attitudes, behaviours and practices towards diabetes mellitus in Kuwait. East Mediterr Health J. 2018;24(11):1098-1102. DOl:https://doi. org/10.26719/2018.24.11.1098

30. Jalilian H, Pezeshki MZ, Janati A, Najafipour F, Imani A, Zozani MA et all. Readiness for diet change and its association with diet knowledge and skills, diet decision making and diet barriers in type 2 diabetic patients. Diabetes \& Metabolic Syndrome: Clinical Research \& Reviews; 2019: 13(5), 2933-8. DOl:https://doi.org/10.1016/j.dsx.2019.07.065

31. Greene G, Ruggiero L, Rossi S, Rossi J. Stage of change for dietary fat reduction in persons with diabetes. J Am Diet Assoc 1997;97(9):A24 DOl:https://doi.org/10.1016/S0002-8223(97)00404-5

32. Bawadi HA, Banks AD, Ammari F, Tayyem RF, Jebreen S. Stage of change of 6 health-related behaviors among patients with type 2 diabetes. Prim Care Diabetes 2012;6(4):319-27. DOI:https://doi.org/10.1016/j.pcd.2012.07.003

33. Holmen $\mathrm{H}$, Wahl A, Torbjørnsen A, Jenum AK, Småstuen MC, Ribu L. Stages of change for physical activity and dietary habits in persons with type 2 diabetes included in a mobile health intervention: the Norwegian study. BMJ Open Diabetes Res Care. 2016; 4(1): e000193. DOl:https://dx.doi. org/10.1136\%2Fbmjdrc-2016-000193 